BU-HEP 93-28

December 1993

\title{
Chiral Perturbation Theory on the Lattice; Strong Coupling Expansion
}

\author{
Stanley Myint and Claudio RebBi \\ Physics Department \\ BOSTON UNIVERSITY \\ 590 Commonwealth Avenue \\ Boston, MA 02215, USA \\ submitted to Nuclear Physics B
}

\begin{abstract}
We evaluate the coefficients of the effective chiral Lagrangian to $O\left(p^{4}\right)$ in the strong coupling, large- $\mathrm{N}$ expansion. In this limit we explicitly perform the functional integral over fundamental degrees of freedom and obtain the effective chiral Lagrangian. We perform this calculation on the Body Centered Hypercubical lattice which preserves Euclidean invariance to order $p^{4}$. We further discuss how the coefficients could be obtained numerically, out of the strong-coupling domain.
\end{abstract}

\footnotetext{
${ }^{1}$ This work was supported in part under DOE grant DE-FG02-91ER40676, DOE contract DE-AC0289ER40509, NSF contract PHY-9057173, and by funds from the Texas National Research Laboratory Commission under grant RGFY91B6.
} 


\section{Introduction}

Effective chiral Lagrangians, originally introduced by Weinberg [1], provide a very powerful method to describe the low energy phenomenology of QCD, exploiting the consequences of chiral symmetry and current algebra [2]. The idea underlying effective Lagrangians is simple and elegant. There are many phenomena which, although the fundamental theory involves several different scales of length and energy, only lead to the excitation of a subset of all the degrees of freedom. We will call such degrees of freedom the effective degrees of freedom. Under these circumstances it is conceptually possible to integrate out of the theory all of the other degrees of freedom and describe the phenomena in terms of an alternative Lagrangian, namely the effective Lagrangian, more amenable to calculations. Thus in low energy QCD, below the threshold for production of resonances, the effective degrees of freedom are those of the pseudoscalar fields, i.e. the would be massless Goldstone bosons of spontaneously broken

chiral symmetry, and pion and kaon interactions can be described in terms of an effective chiral Lagrangian. Another example is found in the physics of heavy quarks, where effective non-relativistic Lagrangians can be used to describe a large class of processes which do not lead to the excitation of the relativistic degrees of freedom of the heavy quarks.

The predictive power of effective Lagrangians in general derives from the existence of some underlying exact or approximate symmetry, such as chiral symmetry in QCD, which restricts substantially the type of couplings that can appear in the Lagrangian itself. Moreover, these couplings can be arranged in an expansion into powers of some parameter related to the suppression of the non-effective degrees of freedom (e.g. the magnitude of the external momenta in QCD) and at any order in the expansion there is only a finite number of possible coupling terms. In typical phenomenological applications, then, the corresponding coupling constants are fixed by comparing the results that follow from the effective theory to a subset of the experimental data. This determines the effective Lagrangian, which can subsequently be used to calculate a variety of other experimental observables [3, 4].

Although this way of proceeding provides a very powerful computational tool, capable of producing a large number of theoretical predictions starting from general symmetry considerations and a limited amount of experimental information, it would be even more valuable to be able to calculate the coefficients of the effective Lagrangian directly from first principles. In the case of low energy QCD, this goal appears within reach of advanced lattice numerical simulations and in this article we wish to present a framework by which it can be achieved.

In some sense, once one has the possibility of calculating observables by means of numerical simulation methods, deriving the coefficients of the effective Lagrangian might appear straightforward: one would just need to compare a sufficient number of predictions from the effective Lagrangian to the results of numerical calculations, very much as one does comparing them to experimental data in the phenomenological derivation. But then, from this point of view, why resort to an effective Lagrangian at all, if the observables can be calculated directly? The point is that numerical simulations provide only an approximate computational tool, where the accuracy of the calculations is severely limited by statistical errors and the discretization procedure. The precision by which one can determine quantities such as the coefficients of an effective chiral Lagrangian, or even the feasibility of such a calculation in absolute, depends dramatically on the choice of a sound computational technique. 
The basic idea underlying the method we wish to propose is that the separation into effective and non-effective degrees of freedom of the continuum theory will be mirrored by a similar distinction in the theory discretized over a lattice. It will then be possible to define a lattice effective Lagrangian to be used as an intermediate step in the derivation of the coefficients of the continuum chiral Lagrangian from the fundamental theory discretized over the lattice. The advantage of this two step procedure is that the lattice effective Lagrangian will contain explicitly the collective fields responsible for the long distance behavior of the fundamental lattice theory. The calculation of its coefficients, which for realistic applications will have to be made through a numerical integration of the non-effective degrees of freedom, will then only involve shorter scales of length, and should be feasible on a lattice of moderate size. Being able to reduce the size of the lattice, and therefore the total number of variables in a numerical simulation, produces major advantages for the accuracy and efficiency of the calculation.

In this article we will illustrate these ideas by deriving the lattice effective chiral Lagrangian in the strong coupling approximation and using it to calculate, always for strong coupling, the coefficients of the $p^{4}$ terms of the continuum Lagrangian. This will be done in Section 3, after a quick recapitulation, presented in Section 2, of the form of the continuum chiral Lagrangian and of some features of the lattice discretization of the QCD fundamental Lagrangian that will be relevant for the rest of the paper. Section 3 owes a lot to the truly pioneering works of Kluberg-Stern, Morel, Napoly and Peterson [5] and of Kawamoto and Smit [6] where the methods for integrating over quark and gauge degrees of freedom in the strong coupling limit were clearly laid out and an effective strong coupling Lagrangian, expressed in terms of meson fields, was first derived. We add to their work, however, the consideration of a different lattice, namely the Body Centered Hypercubical (BCH) or F4 lattice, whose greater symmetry implies that invariance under lattice transformations carries over to Euclidean invariance up to the terms of order $p^{4}$ in the continuum. This is clearly crucial for a strong coupling derivation of the $p^{4}$ terms in the effective chiral Lagrangian. We were inspired to use this lattice by a talk presented by Boghosian at the Amsterdam Symposium on Lattice Gauge Theories. This talk [7] focused on the application of the BCH lattice in the theory of cellular automata and lattice gases, however the $p^{4}$ invariance had been previously emphasized and exploited by Neuberger [8] in a study of the Higgs phenomenon. In the context of lattice field theories, this lattice was also considered in references [9] -[11].

Finally, in Section 4, we will discuss how one could derive the parameters of the chiral lattice Lagrangian for more realistic values of the QCD coupling constant, closer to the continuum limit, by numerical simulations coupled to real space renormalization techniques.

\section{Chiral Perturbation Theory and Lattice QCD}

For the convenience of the reader and in order to establish a point of reference we review the basic features of chiral perturbation theoryf and of the lattice formulation of QCD.

Following Gasser and Leutwyler [3] and [4], we introduce the generating functional for the connected Green's functions of QCD in the presence of scalar, pseudoscalar, vector and

\footnotetext{
${ }^{2}$ Several reviews are given in references [12] - 14].
} 
axial vector sources ${ }^{3}$

$$
\begin{gathered}
e^{i W[s, p, v, a]}=<0\left|T e^{i \int d^{4} x \mathcal{L}}\right| 0> \\
\mathcal{L}=\mathcal{L}_{Q C D}^{0}-\bar{q}(x)\left[s(x)-i \gamma_{5} p(x)\right] q(x)+\bar{q}(x) \gamma^{\mu}\left[v_{\mu}(x)+\gamma_{5} a_{\mu}(x)\right] q(x) .
\end{gathered}
$$

In this expression, $q, \bar{q}$ represent the lightest quark flavor triplet and $\mathcal{L}_{Q C D}^{0}$ is what remains of the Lagrangian of QCD when the masses of three lightest quarks are set to zero

$$
\mathcal{L}_{Q C D}^{0}=-\frac{1}{4 g^{2}} G_{\mu \nu}(x) G^{\mu \nu}(x)+\bar{q}(x) \gamma^{\mu}\left[i \partial_{\mu}+G_{\mu}(x)\right] q(x) .
$$

$s(x), p(x), v_{\mu}(x)$ and $a_{\mu}(x)$ are Hermitian matrices in flavor space. The quark mass matrix

$$
\mathcal{M}=\left(\begin{array}{lll}
m_{u} & & \\
& m_{d} & \\
& & m_{s}
\end{array}\right)
$$

is contained in the scalar field $s(x)$. In this paper we will not consider the $S U(3)$ singlet vector and axial vector current:

$$
\operatorname{Tr}\left[v_{\mu}(x)\right]=\operatorname{Tr}\left[a_{\mu}(x)\right]=0 .
$$

It is convenient to define the right and left handed currents:

$$
r_{\mu}(x) \equiv v_{\mu}(x)+a_{\mu}(x), \quad l_{\mu}(x) \equiv v_{\mu}(x)-a_{\mu}(x)
$$

The Lagrangian (2) exhibits a local flavor chiral symmetry $S U_{L}(3) \times S U_{R}(3)$ :

$$
\begin{aligned}
q(x) & \rightarrow L(x) \frac{1-\gamma_{5}}{2} q(x)+R(x) \frac{1+\gamma_{5}}{2} q(x), \\
r_{\mu}(x) & \rightarrow R(x) r_{\mu}(x) R(x)^{\dagger}+i R(x) \partial_{\mu} R(x)^{\dagger}, \\
l_{\mu}(x) & \rightarrow L(x) l_{\mu}(x) L(x)^{\dagger}+i L(x) \partial_{\mu} L(x)^{\dagger}, \\
s(x)+i p(x) & \rightarrow R(x)[s(x)+i p(x)] L(x)^{\dagger},
\end{aligned}
$$

with matrices $L(x), R(x) \in S U(3)$.

The goal of chiral perturbation theory is to produce an expansion of the generating functional $W$ in powers of the external momenta and quark masses. Because of the presence of pseudo Goldstone bosons one can not simply perform a Taylor series expansion of $W$, but one must introduce suitable fields to account for their degrees of freedom. These fields are collected in a unitary $3 \times 3$ matrix:

$$
U(x)=\exp \left[\frac{i \pi^{a}(x) \lambda^{a}}{F^{0}}\right], \quad U(x) U(x)^{\dagger}=1 .
$$

$\lambda^{a}, a=1, \ldots, 8$ are Hermitian generators of $S U(3)$, with the normalization

$$
\operatorname{Tr}\left(\lambda^{a} \lambda^{b}\right)=2 \delta^{a b}
$$

\footnotetext{
${ }^{3}$ Although it is in principle possible to include tensor sources, they are usually neglected.
} 
$\pi^{a}(x)$ are real pseudoscalar fields and $F^{0}$ is the pion decay constant in the chiral limit:

$$
\begin{aligned}
<0\left|\bar{q}(x) \gamma_{\mu} \gamma_{5} \frac{\lambda^{a}}{2} q(x)\right| \pi^{b}> & =i f_{\pi} p_{\mu} \delta^{a b}, \\
f_{\pi}=F_{0}\left(1+O\left(m_{q}\right)\right) & \approx 93.3 \mathrm{MeV} .
\end{aligned}
$$

To the lowest order $O\left(p^{2}\right)$, one must only evaluate tree level diagrams with a classical Lagrangian involving terms with two derivatives and one power of scalar and pseudoscalar sources:

$$
\mathcal{L}_{2}=\frac{F_{0}^{2}}{4} \operatorname{Tr}\left\{\left[D_{\mu} U(x)\right]\left[D^{\mu} U(x)\right]^{\dagger}+\chi(x) U(x)^{\dagger}+\chi(x)^{\dagger} U(x)\right\} .
$$

In this expression,

$$
D_{\mu} U(x)=\partial_{\mu} U(x)-i r_{\mu}(x) U(x)+i U(x) l_{\mu}(x)
$$

is a flavor covariant derivative and matrix $\chi(x)$ collects the scalar and pseudoscalar fields:

$$
\chi(x)=2 B_{0}[s(x)+i p(x)] .
$$

The constant $B_{0}$ is related to the quark condensate in the chiral limit:

$$
<0|\bar{u} u| 0>_{0}=<0|\bar{d} d| 0>_{0}=<0|\bar{s} s| 0>_{0}=-F_{0}^{2} B_{0}\left(1+O\left(m_{q}\right)\right) .
$$

In the expansion in powers of pion momenta, vector and axial vector sources count as contributions of order $p$, whereas scalar and pseudoscalar sources count as contributions of order $p^{2}$.

To the next to leading order $O\left(p^{4}\right)$, one encounters two kinds of contributions:

- one loop diagrams with $\mathcal{L}_{2}$ vertices;

- tree diagrams with $\mathcal{L}_{4}$ vertices.

$\mathcal{L}_{4}$ is the most general Lagrangian which contains four powers of momenta consistent with the local chiral flavor symmetry (7). Therefore, it can be formed only from the covariant derivatives (12) and the field strengths for right and left-handed fields:

$$
\begin{gathered}
F_{R}^{\mu \nu}=\partial^{\mu} r^{\nu}-\partial^{\nu} r^{\mu}-i\left[r^{\mu}, r^{\nu}\right], \\
F_{L}^{\mu \nu}=\partial^{\mu} l^{\nu}-\partial^{\nu} l^{\mu}-i\left[l^{\mu}, l^{\nu}\right] . \\
\mathcal{L}_{4}=L_{1}\left\{\operatorname{Tr}\left[\left(D_{\mu} U\right)\left(D^{\mu} U\right)^{\dagger}\right]\right\}^{2}+L_{2} \operatorname{Tr}\left[\left(D_{\mu} U\right)^{\dagger}\left(D_{\nu} U\right)\right] \operatorname{Tr}\left[\left(D^{\mu} U\right)^{\dagger}\left(D^{\nu} U\right)\right] \\
+L_{3} \operatorname{Tr}\left[\left(D_{\mu} U\right)^{\dagger}\left(D^{\mu} U\right)\left(D_{\nu} U\right)^{\dagger}\left(D^{\nu} U\right)\right]+L_{4} \operatorname{Tr}\left[\left(D_{\mu} U\right)^{\dagger}\left(D^{\mu} U\right)\right] \operatorname{Tr}\left(\chi^{\dagger} U+\chi U^{\dagger}\right) \\
+L_{5} \operatorname{Tr}\left[\left(D_{\mu} U\right)^{\dagger}\left(D^{\mu} U\right)\left(\chi^{\dagger} U+\chi U^{\dagger}\right)\right]+L_{6}\left[\operatorname{Tr}\left(\chi^{\dagger} U+\chi U^{\dagger}\right)\right]^{2} \\
+L_{7}\left[\operatorname{Tr}\left(\chi^{\dagger} U-\chi U^{\dagger}\right)\right]^{2}+L_{8} \operatorname{Tr}\left(\chi^{\dagger} U \chi^{\dagger} U+\chi U^{\dagger} \chi U^{\dagger}\right) \\
-i L_{9} \operatorname{Tr}\left[F_{\mu \nu}^{R}\left(D^{\mu} U\right)\left(D^{\nu} U\right)^{\dagger}+F_{\mu \nu}^{L}\left(D^{\mu} U\right)^{\dagger}\left(D^{\nu} U\right)\right]+L_{10} \operatorname{Tr}\left(U^{\dagger} F_{\mu \nu}^{R} U F^{L \mu \nu}\right) \\
+H_{1} \operatorname{Tr}\left(F_{\mu \nu}^{R} F^{R \mu \nu}+F_{\mu \nu}^{L} F^{L \mu \nu}\right)+H_{2} \operatorname{Tr}\left(\chi^{\dagger} \chi\right) .
\end{gathered}
$$

As we see, at leading order, chiral symmetry restricts the number of terms in the effective chiral Lagrangian to only two, so that one needs to know only two constants, $F_{0}$ and $B_{0}$, in 
order to describe all low energy phenomena in QCD to order $p^{2}$. At the next to leading order one needs ten more constants: $L_{1}-L_{10}$. (The terms with coefficients $H_{1}$ and $H_{2}$ are of no physical significance and we will disregard them from now on.)

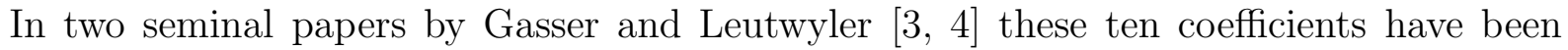
calculated to reasonable accuracy by comparison with experimental data. It would be of course important to be able to determine all the coefficients theoretically. This requires a non-perturbative calculation and lattice numerical simulations represent today the only method by which such a calculation could be carried out from first principles. Otherwise one can try to calculate the coefficients from models which are similar to, but easier to solve than QCD. For an account of various attempts at a theoretical determination of the coefficients see, for example, [15, 16, 17] and references therein. In recent years, a rather exhaustive calculation has appeared in references [18, 19].

Our goal is to provide a framework by which the coefficients of the terms of order $p^{4}$ in the chiral Lagrangian can be efficiently calculated by means of a lattice numerical simulation. The idea is to proceed through the intermediary of an effective chiral Lagrangian defined over the lattice. The problem then splits into two separate calculations:

- A numerical simulation is used to calculate the coefficients of the lattice chiral Lagrangian through a matching of lattice observables. As we will argue in Sect. 4, it should be possible to carry out this step on a reasonably small lattice and, therefore, with high statistical accuracy.

- The coefficients of the continuum chiral Lagrangian will be obtained from those of the lattice effective Lagrangian by an expansion of the observables for small lattice momenta and by carrying out the appropriate renormalizations. This step can be performed analytically, by a perturbative expansion of the lattice chiral Lagrangian, if the coefficients, as determined in the previous step, are such as to validate the expansion. Otherwise it would have have to be done numerically.

We believe that one can carry out these two steps with better accuracy than a direct determination of the coefficients of the continuum Lagrangian through a comparison with observables calculated over the lattice.

While in Sect. 4 we plan to discuss in some detail the procedures that could be followed to perform such calculations in the physically interesting domain where continuum scaling sets in, the main result which we wish to present in this paper is an illustration of the method in the strong coupling approximation. Precisely, we wish to establish the possibility of defining a lattice chiral Lagrangian by deriving it explicitly at leading order in the strong coupling expansion in $\beta=6 / g_{0}^{2}$. An expansion for low lattice momenta will then allow us to identify the values of the coefficients of the continuum Lagrangian.

In the balance of this section we review a few basic features of the lattice regularization of QCD that will be useful for our subsequent derivation of the lattice chiral Lagrangian and will also serve to establish our notation.

In the lattice regularization of QCD, Euclidean space-time is generally discretized by the introduction of a regular lattice of points and oriented links joining neighboring points. In the vast majority of applications this lattice is a hypercubical lattice, however, for reasons 
that will become clear in Sect. 3, we will need to work with a different lattice to carry out the strong coupling expansion. Therefore we will leave the detailed geometry of the lattice unspecified for now, but we will assume that the lattice is regular and uniform, with lattice spacing $a$.

We will denote by $x$ the coordinates of the lattice points and by $v$ the fundamental displacement vectors in the lattice. Thus, for clarification, with a hypercubical lattice we would have $x=\left(i_{1} a, i_{2} a, i_{3} a, i_{4} a\right)$ with integer $i_{1}, i_{2}, i_{3}, i_{4}$ and $v$ would range over $(a, 0,0,0),(0, a, 0,0),(0,0, a, 0),(0,0,0, a)$.

The fundamental variables are the gluon fields and the quark fields. The gluon fields are finite elements of color $S U(3)$, which are associated to the oriented links of the lattice. It is rather standard to denote such variables by $U_{x, v}$, but eventually, following another standard notation, we will want to reserve the $U$ symbol for the chiral fields. We will therefore denote the lattice gauge fields by $G_{x, v}$.

The gauge part of the action is constructed from the plaquette variables, i.e. the transport factors around the elementary closed contours of the lattice. The plaquettes are defined by two displacement vectors, and so we will use the notation $G_{x, v_{1} v_{2}}$ to denote a plaquette variable. (For the hypercubical lattice $G_{x, v_{1} v_{2}}=G_{x, v_{1}} G_{x+v_{1}, v_{2}} G_{x+v_{2}, v_{1}}^{\dagger} G_{x, v_{2}}^{\dagger}$ ). The gauge part of the action is then given by

$$
S_{g}=\beta K_{1} \sum_{x, v_{1}, v_{2}} \operatorname{Tr}\left(I-G_{x, v_{1} v_{2}}\right)
$$

where we are summing over all plaquettes with both orientations, $K_{1}$, as well as $K_{2}$ in the equation below, are lattice dependent normalization factors, f and the coupling parameter $\beta$ is related to the bare coupling constant by $\beta=6 / g_{0}^{2}$.

The quark and antiquark fields, 回 $\psi_{x}, \bar{\psi}_{x}$ are defined over the sites of the lattice. The interaction term between quarks and gluons is given by:

$$
S_{\psi}=a^{3} K_{2} \sum_{x} \sum_{v}\left(\bar{\psi}_{x} \psi G_{x, v} \psi_{x+v}-\bar{\psi}_{x+v} \psi G_{x, v}^{\dagger} \psi_{x}\right)
$$

We will also include scalar and pseudo-scalar source terms through a matrix $j_{x}$ :

$$
S_{j}=a^{4} \sum_{x} \bar{\psi}_{x} j_{x} \psi_{x}=a^{4} \sum_{x} \bar{\psi}_{x}\left(s_{x}-i \gamma_{5} p_{x}\right) \psi_{x}
$$

To summarize, equations (17-19) define the QCD action on any regular uniform lattice.

\section{Strong coupling expansion on the $\mathrm{BCH}$ Lattice}

In order to derive a lattice chiral Lagrangian one must integrate out the high energy degrees of freedom, i.e. the quantum fluctuations of the gauge and quark fields, re-expressing the generating functional in terms of pseudoscalar fields conjugate to the external sources. Thus one effectively performs a bosonization of the original theory. For general values of the

\footnotetext{
${ }^{4} K_{1}=K_{2}=1 / 2$ for the hypercubical lattice.

${ }^{5}$ Color, spin and flavor indices will be left implicit where it doesn't affect the clarity of the formula.
} 
bare lattice coupling constant this can only be done numerically, but in the strong coupling and in the large $N$ (number of colors) limits the bosonization can be done analytically. The techniques for deriving an effective Lagrangian in the strong coupling limit were laid down in papers by Kluberg-Stern, Morel, Napoly and Petersson [5] and Kawamoto and Smit [6]. We add, however, to the work of these authors an ingredient that plays a crucial role for the possibility of obtaining the terms of order $p^{4}$ in the chiral Lagrangian. As in most lattice calculations, [5, 6] deal with a hypercubical (HC) lattice. While such a lattice obviously does not have the symmetry of the continuum, all tensors of rank up to two symmetric under the group of lattice transformations are also symmetric under the full group of 4-dimensional rotations of Euclidean space-time. However, this property does not carry through to tensors of higher order, so that, although one could expand the chiral Lagrangian derived on a HC lattice to terms of $4^{\text {th }}$ order in lattice momentum, one would obtain terms that cannot be identified with continuum counterparts. This precludes the possibility of recognizing the structure of the continuum chiral Lagrangian to $O\left(p^{4}\right)$ on the HC lattice.

In order to pursue our calculation, we will study QCD on a different lattice, namely the Body Centered Hypercubical (BCH) or F4 lattice, which, as already mentioned in the introduction, has a remarkable property that not only tensors of rank two but also those of rank four have the continuum symmetry.

The $\mathrm{BCH}$ lattice can be formed in two equivalent ways. One is to visualize it as the usual $\mathrm{HC}$ lattice from which all the sites with $\sum_{\mu} x_{\mu}=$ odd (or even) have been removed. An equivalent way is to take all the sites of the HC lattice together with centers of its elementary cells (hence the name BCH) to represent the sites of the new lattice. The lattice formed in such a way has the largest symmetry group of all four dimensional regular lattices. Whereas the usual HC lattice has 384 element symmetry group, the symmetry group of the $\mathrm{BCH}$ lattice has 1152 elements. A consequence of this is the required $p^{4}$ invariance.

On the $\mathrm{BCH}$ lattice every site has 24 nearest neighbors forming triangular plaquettes. In terms of unit vectors of the hypercubical lattice $\vec{e}_{i}$, the links in the positive direction $v_{i j}^{\alpha}$ are given by the following twelve linear combinations:

$$
v_{i j}^{\alpha}=\frac{\vec{e}_{i}+\alpha \vec{e}_{j}}{\sqrt{2}},\{1 \leq i<j \leq 4, \alpha= \pm 1\}
$$

The use of the $\mathrm{BCH}$ lattice entails another advantage in the strong coupling limit. It is of rather technical nature and so we will mention it only briefly. The definition of lattice fermions encounters some notorious problems. A straightforward discretization of the Dirac operator leads to the introduction of additional poles in the propagators of the fermions at the corners of the Brillouin zone (species doubling). Although these extra fermionic modes would in general be coupled through the gluonic quantum fluctuations, on a hypercubical lattice there is a fourfold degeneracy of totally decoupled fermionic degrees of freedom. This is akin to starting with one flavor of fermions, but discovering that the theory actually contains four decoupled flavors. As a consequence, the spontaneous breaking of chiral symmetry gives origin to 16 pseudo Goldstone bosons, even though one started with a single flavor. On a $\mathrm{BCH}$ lattice, because of the higher coordination number of its vertices, such a separation of the fermionic fields into four decoupled components (which is not demanded by any general theorem on lattice fermions) does not take place and one maintains the relationship between 
the number of explicit flavor indices and the number of pseudo Goldstone bosons proper of the continuum theory.

In order to study interactions with external vector and axial vector fields, we will introduce traceless Hermitian sources on the positive link $v$ starting from a lattice point $x$ :

$$
\tilde{v}_{x, v}=v_{x, v}^{a} \lambda^{a}, \quad \tilde{a}_{x, v}=a_{x, v}^{a} \lambda^{a} .
$$

We couple these sources to quark and gluon fields in a nonlinear way through an auxiliary field $Y(\tilde{v}, \tilde{a})$ :

$$
Y(\tilde{v}, \tilde{a})_{x, v}=\exp \left\{-\frac{i a}{2}\left[\tilde{v}_{x, v}+\tilde{v}_{x+v, v}+\gamma_{5}\left(\tilde{a}_{x, v}+\tilde{a}_{x+v, v}\right)\right]\right\} .
$$

In the classical continuum limit we require that:

$$
\tilde{v}_{x, v} \rightarrow \frac{\tilde{v}_{i}+\alpha \tilde{v}_{j}}{\sqrt{2}}, \quad \tilde{a}_{x, v} \rightarrow \frac{\tilde{a}_{i}+\alpha \tilde{a}_{j}}{\sqrt{2}}
$$

The interaction term with couplings to external vector and axial vector sources is given by:

$$
S_{\psi}=a^{3} K_{2} \sum_{x} \sum_{v}\left(\bar{\psi}_{x} \psi G_{x, v} Y_{x, v} \psi_{x+v}-\bar{\psi}_{x+v} \psi G_{x, v}^{\dagger} Y_{x, v}^{\dagger} \psi_{x}\right)
$$

Our final expression for the lattice action is:

$$
S=S_{g}+S_{\psi}+S_{j},
$$

with $S_{g}, S_{\psi}$ and $S_{j}$ given in (17), (24) and (19).

We set $K_{1}=K_{2}=1 / 12$ and in the limit of vanishing lattice spacing $a \rightarrow 0$ identify the gauge variables with continuum transport factors through $G_{x, v}=\exp \left[i g_{0} A_{\mu}(x) v^{\mu}\right]$. With this choice, the action (25) has a correct classical continuum limit.

It is worthwhile noting that our action possesses chiral flavor gauge symmetry under transformations:

$$
\begin{aligned}
\psi_{x} & \rightarrow\left(L_{x} \frac{1-\gamma_{5}}{2}+R_{x} \frac{1+\gamma_{5}}{2}\right) \psi_{x}, \\
\bar{\psi}_{x} & \rightarrow \bar{\psi}_{x}\left(L_{x}^{\dagger} \frac{1+\gamma_{5}}{2}+R_{x}^{\dagger} \frac{1-\gamma_{5}}{2}\right), \\
Y_{x, v} & \rightarrow\left(L_{x} \frac{1-\gamma_{5}}{2}+R_{x} \frac{1+\gamma_{5}}{2}\right) Y_{x, v}\left(L_{x+v}^{\dagger} \frac{1-\gamma_{5}}{2}+R_{x+v}^{\dagger} \frac{1+\gamma_{5}}{2}\right), \\
s_{x}+i p_{x} & \rightarrow R_{x}\left(s_{x}+i p_{x}\right) L_{x}^{\dagger},
\end{aligned}
$$

which reduces to the form (7) in the classical continuum limit.

Generating functional on the lattice can be written as a path integral over the quark and gluon fields:

$$
Z(s, p, v, a)=\int D \psi D \bar{\psi} D G e^{-S(\psi, \bar{\psi}, G ; s, p, v, a)} .
$$

\footnotetext{
${ }^{6}$ It has been argued in Ref. [10 that the use of the BCH lattice actually worsens the problem of lattice fermions as one tries to recover the continuum limit. This is irrelevant for our purposes, because our motivation for its use comes solely from the need of obtaining better symmetry properties in the strong coupling limit. We would not advocate using the $\mathrm{BCH}$ lattice in numerical simulations done in the scaling regime, where there is good evidence for the recovery of rotational symmetry with the ordinary HC lattice.
} 
In this expression, $s, p, v, a$ are external sources defined earlier, the action $S$ is given in (25) and the integration is performed over all the lattice variables (for example: $D G \equiv \prod_{x, v} d G_{x, v}$ ).

In the strong coupling expansion, the gauge term (17) is suppressed by $1 / g_{0}^{2}$. Therefore, in the leading order we can neglect it, and the action reduces to $S=S_{\psi}+S_{j}$. Now the gauge variables appear only in $S_{\psi}$, moreover they appear only linearly. This implies that the product of integrals over gauge variables $D G$ factors into a product of one-link integrals:

$$
Z=\int D \psi D \bar{\psi} e^{-S_{j}} \prod_{x, v} \int d G_{x, v} e^{-W_{x, v}}
$$

Here we have defined:

$$
W_{x, v}=\frac{a^{3}}{12}\left(\bar{\psi}_{x} \psi G_{x, v} Y_{x, v} \psi_{x+v}-\bar{\psi}_{x+v} \psi G_{x, v}^{\dagger} Y_{x, v}^{\dagger} \psi_{x}\right)
$$

One link integrals of this type have been calculated for the case when the gauge field couples to a bosonic source in [20]. In [5] it was shown that this result applies equally to an interaction of gauge with fermionic fields. The interested reader should consult reference [5] for the details. It is important to mention that the bosonization procedure can be extended to the next order in $1 / g_{0}^{2}$ expansion [21] and to arbitrary (odd) $N$ [22]. Here we just give the final result for the generating functional to the leading order in the strong coupling, large-N limit:

$$
Z=\int D \psi D \bar{\psi} e^{N \sum_{x, v} \operatorname{Tr}[F(\lambda(x, v))]-S_{j}} .
$$

$\lambda(x, v)$ is a matrix in Dirac-flavor space, defined by its elements]:

$$
\lambda(x, v)_{\alpha \beta}=\frac{a^{6}}{36 N^{2}} \sum_{i, j=1}^{N} \sum_{\delta, \gamma, \epsilon=1}^{4 n}\left[\bar{\psi}_{i}^{\alpha}(x) \psi_{i}^{\delta}(x)\left(\psi Y_{x, v}^{\dagger}\right)_{\gamma \delta} \bar{\psi}_{j}^{\gamma}(x+v) \psi_{j}^{\epsilon}(x+v)\left(\psi Y_{x, v}\right)_{\beta \epsilon}\right] .
$$

$F(\lambda)$ is the following matrix function of $\lambda$ :

$$
F(\lambda)=1-\sqrt{1-\lambda}+\ln \frac{1+\sqrt{1-\lambda}}{2}
$$

Next we proceed to convert the fermionic path integral into one over bosonic variables as in references [5], [6]. In the expression (30), $N \operatorname{Tr}[F(\lambda)]$ is the interaction term. It is important to notice that it depends only on color singlet combinations $\bar{\psi}_{i}^{\alpha}(x) \psi_{i}^{\delta}(x)$ at different points on the lattice. Since these appear also in the source term $S_{j}$, we can write:

$$
\frac{a^{3}}{N} \sum_{i}\left[\bar{\psi}_{i}^{\alpha}(x) \psi_{i}^{\delta}(x)\right]=\frac{1}{N a} \frac{\partial}{\partial j_{x}^{\alpha \delta}}\left[a^{4} \bar{\psi}_{x}^{\alpha} j_{x}^{\alpha \delta} \psi_{x}^{\delta}\right]=\frac{1}{N a} \frac{\partial S_{j}}{\partial j_{x}^{\alpha \delta}} .
$$

Therefore we can rewrite the full generating functional as:

$$
Z=e^{N \sum_{x, v} \operatorname{Tr}[F(\lambda(x, v))]} \int D \psi D \bar{\psi} e^{-S_{j}},
$$

\footnotetext{
${ }^{7}$ Greek letters will denote indices in the direct product of Dirac and flavor spaces, and Latin letters the color indices. In this section we will take the number of flavors to be $n$ and only at the end specialize to $n=3$.

${ }^{8} F(\lambda)$ should be understood as a power series in Grassmann variables.
} 
with:

$$
\lambda(x, v)_{\alpha \beta}=\frac{1}{36(N a)^{2}} \sum_{\delta, \gamma, \epsilon=1}^{4 n}\left[\frac{\partial}{\partial j_{x}^{\alpha \delta}}\left(\psi Y_{x, v}^{\dagger}\right)_{\gamma \delta} \frac{\partial}{\partial j_{x+v}^{\gamma \epsilon}}\left(\psi Y_{x, v}\right)_{\beta \epsilon}\right] .
$$

The path integral over fermionic variables factorizes into a product of one-site integrals:

$$
\begin{gathered}
Z_{0} \equiv \int D \psi D \bar{\psi} e^{-S_{j}}=\prod_{x} \int d \psi_{x} d \bar{\psi}_{x} e^{-a^{4}\left(\bar{\psi}_{x} j_{x} \psi_{x}\right)}=\prod_{x} z_{0}\left(j_{x}\right), \\
z_{0}\left(j_{x}\right) \equiv \int d \psi_{x} d \bar{\psi}_{x} e^{-a^{4}\left(\bar{\psi}_{x} j_{x} \psi_{x}\right)}
\end{gathered}
$$

In [5, 6] it was shown that $z_{0}\left(j_{x}\right)$ can be written as an integral over bosonic variables:

$$
z_{0}\left(j_{x}\right)=\int d \mathcal{M}_{x} e^{N \operatorname{Tr}\left(a j_{x} \mathcal{M}_{x}\right)-N \operatorname{Tr}\left(\ln \mathcal{M}_{x}\right)+\text { const }} .
$$

Here $\mathcal{M}_{x}$ is a unitary bosonic matrix in Dirac-flavor space, the trace is over Dirac-flavor indices and the constant is irrelevant so it will be omitted hereafter.

Using (34) and (35) we obtain an expression for the full generating functional:

$$
Z=\int D \mathcal{M} e^{N\left\{\sum_{x, v} \operatorname{Tr}\left[F\left(\lambda_{x, v}\right)\right]+\sum_{x} \operatorname{Tr}\left(a j_{x} \mathcal{M}_{x}\right)-\sum_{x} \operatorname{Tr}\left(\ln \mathcal{M}_{x}\right)\right\}} \equiv \int D \mathcal{M} e^{-S(\mathcal{M})},
$$

where $\lambda$ is now a bosonic matrix:

$$
\lambda(x, v)=\frac{1}{36} \psi Y_{x, v} \mathcal{M}_{x+v} \psi Y_{x, v}^{\dagger} \mathcal{M}_{x}
$$

The transformation of $\mathcal{M}$ under chiral gauge symmetry follows from (26):

$$
\mathcal{M}_{x} \rightarrow\left(L_{x} \frac{1-\gamma_{5}}{2}+R_{x} \frac{1+\gamma_{5}}{2}\right) \mathcal{M}_{x}\left(L_{x}^{\dagger} \frac{1+\gamma_{5}}{2}+R_{x}^{\dagger} \frac{1-\gamma_{5}}{2}\right) .
$$

One can see that this, together with a law of transformation of $Y_{x, v}$ (26) makes the action $S(\mathcal{M})$ chirally gauge invariant. Now we look for a translationally invariant saddle point of the action $S(\mathcal{M})$ of the form:

$$
\mathcal{M}_{x}^{0}=u_{0}
$$

$u_{0}$ being proportional to the unit matrix in Dirac-flavor space. From (40), using the fact that $\psi^{2}=1$, we obtain $\lambda_{0} \equiv \lambda\left(x, u_{0}\right)=u_{0}^{2} / 36$. For a flavor diagonal mass matrix $j_{x}=s_{x}=\bar{m}$ and no other external sources, the action at this point reduces to:

$$
S^{0}=-N \sum_{x, v} \operatorname{Tr}\left[F\left(\frac{u_{0}^{2}}{36}\right)-\frac{1}{12} \ln u_{0}+\frac{1}{12} a \bar{m} u_{0}\right] .
$$

The effective potential is given by:

$$
V_{\text {eff }}=-\frac{S\left(\mathcal{M}_{x}^{0}\right)}{\text { volume }}
$$

By requiring:

$$
\frac{d V_{e f f}}{d u_{0}}=0
$$


we find the saddle point as a positive solution of the quadratic equation:

$$
u_{0}=\frac{-22(a \bar{m}) \pm \sqrt{(24 a \bar{m})^{2}+1472}}{16+(a \bar{m})^{2}}
$$

For the massless case $u_{0}=\sqrt{23} / 2 \approx 2.40$.

We will parameterize the field $\mathcal{M}_{x}$ in a nonlinear way [6]:

$$
\mathcal{M}_{x}=u_{0} \exp \left[\frac{i}{F_{0}}\left(S_{x}+\pi_{x} \gamma_{5}+V_{x}^{\nu} \gamma_{\nu}+i A_{x}^{\nu} \gamma_{\nu} \gamma_{5}+\frac{1}{2} T_{x}^{\nu \rho} \sigma_{\nu \rho}\right)\right]
$$

Here $S, \pi, V^{\nu}, A^{\nu}$ and $T^{\nu \rho}$ are sixteen Hermitian matrices in flavor space. Of these fields, only $\pi$ are real Goldstone bosons, as we will show in appendix A.

In principle, we need to integrate out the scalar, vector, axial vector and tensor fields in order to obtain an effective action for pseudoscalar mesons. In the first approximation we will neglect these contributions? and concentrate only on the direct interactions among Goldstone bosons:"ण0

$$
\mathcal{M}_{x}=u_{0} \exp \left(i \frac{\pi_{x} \gamma_{5}}{F_{0}}\right)
$$

Next we expand the action $S(\mathcal{M})$ up to fourth order in Taylor series around the vacuum (42) and (46). This is sufficient to extract coefficients of effective chiral Lagrangian to order $p^{4}$ since the deviation from the vacuum $\lambda_{x, v}-\lambda_{0}$ is of $O(p)$.

The action in (39) can be written as:

$$
S=-N \sum_{x, v} \operatorname{Tr}\left[F\left(\lambda_{x, v}\right)\right]-N u_{0} \sum_{x} \operatorname{Tr}\left(a j_{x} \mathcal{M}_{x}\right)
$$

We expand $\operatorname{Tr}\left[F\left(\lambda_{x, v}\right)\right]$ to the fourth order:

$$
\operatorname{Tr}\left[F\left(\lambda_{x, v}\right)\right]=\sum_{n=1}^{4}\left\{\frac{1}{n !}\left(\frac{\partial^{n} F}{\partial \lambda^{n}}\right)_{\lambda_{0}} \operatorname{Tr}\left[\left(\lambda_{x, v}-\lambda_{0}\right)^{n}\right]\right\} .
$$

We evaluate the Dirac traces in a basis where $\gamma_{5}=\operatorname{diag}(1,1,-1,-1)$. By using the relations

$$
\begin{aligned}
\psi \mathcal{M}_{x+v} & =\mathcal{M}_{x+v}^{\dagger} \psi, \\
\psi Y(\tilde{v}, \tilde{a})_{x, v} & =Y(\tilde{v},-\tilde{a})_{x, v} \psi,
\end{aligned}
$$

which follow from definitions of $\mathcal{M}$ and $Y(\tilde{v}, \tilde{a})$, we can take the trace over spin indices to obtain:

$$
\operatorname{Tr}\left[\left(\lambda_{x, v}-\lambda_{0}\right)^{n}\right]=2 \lambda_{0}^{n} \operatorname{Tr}_{f}\left[\left(Z_{x, v} U_{x+v}^{\dagger} W_{x, v}^{\dagger} U_{x}-1\right)^{n}+\text { h.c. }\right] .
$$

\footnotetext{
${ }^{9}$ They are suppressed by the masses of relevant resonances.

${ }^{10}$ In this paper we will not consider the effects of the $U(1)$ anomaly in any detail, so we will restrict our attention to the traceless part of $\pi_{x}$.
} 
Here we have defined new variables which are matrices in flavor space only:

$$
\begin{aligned}
U_{x} & \equiv \exp \left(i \frac{\pi_{x}}{F_{0}}\right) \\
W_{x, v} & \equiv \exp \left\{\frac{-i a}{2}\left[\tilde{v}_{x, v}+\tilde{v}_{x+v, v}+\left(\tilde{a}_{x, v}+\tilde{a}_{x+v, v}\right)\right]\right\} \\
Z_{x, v} & \equiv \exp \left\{\frac{-i a}{2}\left[\tilde{v}_{x, v}+\tilde{v}_{x+v, v}-\left(\tilde{a}_{x, v}+\tilde{a}_{x+v, v}\right)\right]\right\} .
\end{aligned}
$$

$\operatorname{Tr}_{f}$ indicates a trace over flavor indices, and from now on we will omit the subscript $f$. Evaluating the second term in (49) is straightforward and one obtains the expression for the action in terms of the new variables:

$$
\begin{aligned}
S= & -2 N \sum_{x, v} \sum_{n=1}^{4} \frac{\lambda_{0}^{n}}{n !}\left(\frac{\partial^{n} F}{\partial \lambda^{n}}\right)_{\lambda_{0}} \operatorname{Tr}\left[\left(Z_{x, v} U_{x+v}^{\dagger} W_{x, v}^{\dagger} U_{x}-1\right)^{n}+\text { h.c. }\right] \\
& -2 N u_{0} a \sum_{x} \operatorname{Tr}\left[\left(s_{x}-i p_{x}\right) U_{x}+\text { h.c. }\right] .
\end{aligned}
$$

Relations (26), (41) and (53) determine the effect of chiral flavor symmetry transformations on the new variables!:

$$
\begin{aligned}
U_{x} & \rightarrow R_{x} U_{x} L_{x}^{\dagger} \\
W_{x, v} & \rightarrow R_{x} W_{x, v} R_{x+v}^{\dagger} \\
Z_{x, v} & \rightarrow L_{x} Z_{x, v} L_{x+v}^{\dagger}
\end{aligned}
$$

The next step is to re-express $\operatorname{Tr}\left[\left(\lambda_{x, v}-\lambda_{0}\right)^{n}\right]$ in terms of a more convenient variable which will facilitate expansion in powers of momentum (or equivalently powers of lattice spacing a). The new variable is $\alpha$, defined as:

$$
\begin{aligned}
\alpha & \equiv\left(\triangle_{v} U_{x}\right)^{\dagger}\left(\triangle_{v} U_{x}\right) \\
\triangle_{v} U_{x} & \equiv W_{x, v}^{\dagger} U_{x}-U_{x+v} Z_{x, v}^{\dagger}
\end{aligned}
$$

One can express the traces $\operatorname{Tr}\left[\left(\lambda_{x, v}-\lambda_{0}\right)^{n}\right]$ in terms of $\alpha$ :

$$
\begin{aligned}
\operatorname{Tr}\left[\lambda_{x, v}-\lambda_{0}\right] & =-2 \lambda_{0} \operatorname{Tr}(\alpha), \\
\operatorname{Tr}\left[\left(\lambda_{x, v}-\lambda_{0}\right)^{2}\right] & =2 \lambda_{0}^{2}\left[\operatorname{Tr}\left(\alpha^{2}\right)-2 \operatorname{Tr}(\alpha)\right], \\
\operatorname{Tr}\left[\left(\lambda_{x, v}-\lambda_{0}\right)^{3}\right] & =2 \lambda_{0}^{3}\left[3 \operatorname{Tr}\left(\alpha^{2}\right)-\operatorname{Tr}\left(\alpha^{3}\right)\right], \\
\operatorname{Tr}\left[\left(\lambda_{x, v}-\lambda_{0}\right)^{4}\right] & =2 \lambda_{0}^{4}\left[\operatorname{Tr}\left(\alpha^{4}\right)-4 \operatorname{Tr}\left(\alpha^{3}\right)+2 \operatorname{Tr}\left(\alpha^{2}\right)\right] .
\end{aligned}
$$

This is very convenient when one truncates the momentum expansion as we will see in a moment. We will denote by $\triangle_{v}^{i} U_{x}$ the coefficients in the expansion of $\triangle_{v} U_{x}$ in powers of $a$. Relations (53) and (56) imply that the lowest order term in this expansion is the linear term:

$$
\triangle_{v} U_{x} \equiv a\left(\triangle_{v}^{1} U_{x}\right)+\frac{a^{2}}{2}\left(\triangle_{v}^{2} U_{x}\right)+\frac{a^{3}}{6}\left(\triangle_{v}^{3} U_{x}\right)+\ldots
$$

\footnotetext{
${ }^{11}$ Note that the usual transformation law for $U$ fields follows from the initial form of transformation of quark fields (26) and the sequence of changes of variables that we performed.
} 
Since the expansion of $\alpha$ starts with terms which are quadratic in $a$, we can neglect terms proportional to $\operatorname{Tr}\left(\alpha^{3}\right), \operatorname{Tr}\left(\alpha^{4}\right)$ in equations (57), and keep only those proportional to $\operatorname{Tr}(\alpha)$ and $\operatorname{Tr}\left(\alpha^{2}\right)$.

Next we define the right and left handed external sources analogously to the continuum case:

$$
r_{v} \equiv v_{v}+a_{v}, \quad l_{v} \equiv v_{v}-a_{v}
$$

Any variable which under the lattice symmetry transforms in the same way as the link $v_{i j}^{\alpha}$ on which it is defined will from now on be denoted by a subscript $v$. For example:

$$
a_{v} \equiv \frac{a_{i}+\alpha a_{j}}{\sqrt{2}}, \quad a_{v}^{2} \equiv\left(a_{v}\right)^{2}, \text { etc. }
$$

Partial derivatives in the direction of the link $v_{i j}^{\alpha}$ are defined similarly:

$$
\partial_{v} a \equiv \frac{\partial_{i} a+\alpha \partial_{j} a}{\sqrt{2}}, \quad \partial_{v}^{2} a \equiv \partial_{v}\left(\partial_{v} a\right), \text { etc. }
$$

These derivatives appear in the Taylor series expansion of $r_{x+v, v}$ and $R_{x+v}$ in powers of a:

$$
\begin{aligned}
r_{x+v, v} & =r_{v}+a\left(\partial_{v} r_{v}\right)+\frac{a^{2}}{2}\left(\partial_{v}^{2} r_{v}\right)+\ldots \\
R_{x+v} & =R+a\left(\partial_{v} R\right)+\frac{a^{2}}{2}\left(\partial_{v}^{2} R\right)+\ldots
\end{aligned}
$$

and similarly for $l_{x+v, v}$ and $L_{x+v}[$. By substituting the previous Taylor series expansions into equations (53) and (55) one can derive the laws of transformation of $r_{v}$ and $l_{v}$ :

$$
\begin{gathered}
r_{v} \rightarrow R r_{v} R^{\dagger}+i R\left(\partial_{v} R^{\dagger}\right), \\
l_{v} \rightarrow L l_{v} L^{\dagger}+i L\left(\partial_{v} L^{\dagger}\right) .
\end{gathered}
$$

Since this is true for any $v_{i j}^{\alpha}(20)$, in the classical continuum limit (23) this law of transformation becomes the usual law of transformation of gauge fields (7). Let us now define $D_{v} U$ on the link $v$ in analogy with the continuum covariant derivative:

$$
D_{v} U \equiv \partial_{v} U-i r_{v} U+i U l_{v} .
$$

In the classical continuum limit, $D_{v} U$ reduces to its continuum counterpart (12). Moreover, from the transformation laws (63), one can see that $D_{v} U$ transforms covariantly on the lattice:

$$
D_{v} U \rightarrow R\left(D_{v} U\right) L^{\dagger} .
$$

We now expand $\triangle_{v} U_{x}$ in a Taylor series in $a$ by a straightforward but tedious calculation and then re-express coefficients in this expansion, defined in (58), in terms of the lattice covariant derivatives $D_{v} U: \square$

$$
\begin{aligned}
& \triangle_{v}^{1} U_{x}=-D_{v} U \\
& \triangle_{v}^{2} U_{x}=-D_{v}^{2} U-2 i r_{v} D_{v} U \\
& \triangle_{v}^{3} U_{x}=-D_{v}^{3} U-3 i r_{v} D_{v}^{2} U-3 i\left(\partial_{v} r_{v}\right) D_{v} U+3 r_{v}^{2} D_{v} U .
\end{aligned}
$$

\footnotetext{
${ }^{12}$ Here we are using the convention that, when there is no subscript $x$, a variable (or its derivative), is evaluated at $x$.

${ }^{13}$ Here by $D_{v}^{2} U$ we mean $D_{v}\left(D_{v} U\right)$ etc.
} 
Although not all coefficients in the expansion of $\triangle_{v} U$ are covariant under chiral transformations, $\operatorname{Tr}(\alpha)$ and $\operatorname{Tr}\left(\alpha^{2}\right)$ are manifestly covariant!

$$
\begin{gathered}
\operatorname{Tr}(\alpha)=\operatorname{Tr}\left\{a^{2}\left(D_{v} U\right)^{\dagger}\left(D_{v} U\right)+a^{4}\left[\frac{1}{6}\left(D_{v} U\right)^{\dagger}\left(D_{v}^{3} U\right)+\text { h.c. }+\frac{1}{4}\left(D_{v}^{2} U\right)^{\dagger}\left(D_{v}^{2} U\right)\right]\right\}, \\
\operatorname{Tr}\left(\alpha^{2}\right)=\operatorname{Tr}\left[a^{4}\left(D_{v} U\right)^{\dagger}\left(D_{v} U\right)\left(D_{v} U\right)^{\dagger}\left(D_{v} U\right)\right] .
\end{gathered}
$$

In appendix B, we derive results for the summation of tensors of rank two and four over the twelve links in the positive direction:

$$
\begin{gathered}
\sum_{v} a_{v} b_{v}=3 \sum_{\mu} a_{\mu} b^{\mu} \\
\sum_{v} a_{v} b_{v} c_{v} d_{v}=\frac{1}{2} \sum_{\mu, \nu}\left(a_{\mu} b^{\mu} c_{\nu} d^{\nu}+a_{\mu} c_{\nu} b^{\mu} d^{\nu}+a_{\mu} d_{\nu} b^{\nu} c^{\mu}\right) .
\end{gathered}
$$

By using these expressions, summing over links and Wick rotating back to Minkowski space, we get manifestly Lorentz and gauge invariant expressions for the traces:

$$
\begin{aligned}
\sum_{v} \operatorname{Tr}(\alpha)= & \operatorname{Tr}\left\{\frac { 1 } { 1 2 } \left[\left(D_{\mu} U\right)^{\dagger}\left(D^{\mu} D_{\nu} D^{\nu} U\right)+\left(D_{\mu} U\right)^{\dagger}\left(D^{\nu} D^{\mu} D_{\nu} U\right)+\left(D_{\mu} U\right)^{\dagger}\left(D^{\nu} D_{\nu} D^{\mu} U\right)\right.\right. \\
+ \text { h.c }]+ & \left.\frac{1}{8}\left[\left(D^{\mu} D_{\mu} U\right)^{\dagger}\left(D^{\nu} D_{\nu} U\right)+\left(D_{\mu} D_{\nu} U\right)^{\dagger}\left(D^{\mu} D^{\nu} U\right)+\left(D_{\mu} D_{\nu} U\right)^{\dagger}\left(D^{\nu} D^{\mu} U\right)\right]\right\} \\
\sum_{v} \operatorname{Tr}\left(\alpha^{2}\right)= & \frac{1}{2} \operatorname{Tr}\left[\left(D_{\mu} U\right)^{\dagger}\left(D^{\mu} U\right)\left(D_{\nu} U\right)^{\dagger}\left(D^{\nu} U\right)+\left(D_{\mu} U\right)^{\dagger}\left(D_{\nu} U\right)\left(D^{\mu} U\right)^{\dagger}\left(D^{\nu} U\right)\right. \\
& \left.+\left(D_{\mu} U\right)^{\dagger}\left(D_{\nu} U\right)\left(D^{\nu} U\right)^{\dagger}\left(D^{\mu} U\right)\right] .
\end{aligned}
$$

The last step is to substitute these expressions back into (58). The action on the lattice gives the Lagrangian after dividing with the volume of space-time on the BCH lattice:

$$
a^{4} \sum_{x}=2 \int d^{4} x
$$

We can transform all the terms of order $p^{4}$ into the canonical basis of 4 using the property of $\mathrm{SU}(3)$ matrices derived there:

$$
\begin{aligned}
\operatorname{Tr}\left[\left(D_{\mu} U\right)^{\dagger}\left(D_{\nu} U\right)\left(D^{\mu} U\right)^{\dagger}\left(D^{\nu} U\right)\right] & =-2 \operatorname{Tr}\left[\left(D^{\mu} U\right)^{\dagger}\left(D_{\mu} U\right)\left(D^{\nu} U\right)^{\dagger}\left(D_{\nu} U\right)\right] \\
+\frac{1}{2}\left\{\operatorname{Tr}\left[\left(D^{\mu} U\right)^{\dagger}\left(D_{\mu} U\right)\right]\right\}^{2} & +\operatorname{Tr}\left[\left(D^{\mu} U\right)^{\dagger}\left(D^{\nu} U\right)\right] \operatorname{Tr}\left[\left(D_{\mu} U\right)^{\dagger}\left(D_{\nu} U\right)\right]
\end{aligned}
$$

as well as the equation of motion at order $p^{2}$ :

$$
\left(D^{\mu} D_{\mu} U\right) U^{\dagger}-U\left(D^{\mu} D_{\mu} U\right)^{\dagger}=\chi U^{\dagger}-U \chi^{\dagger}-\frac{1}{3} \operatorname{Tr}\left(\chi U^{\dagger}-U \chi^{\dagger}\right) .
$$

We have collected these results in Table 1. and compared them with the coefficients which have been determined experimentally at the scale of $m_{\eta}$ in 4 


\begin{tabular}{|c|r|rll|}
\hline & theory & \multicolumn{3}{|c|}{ experiment } \\
\hline$L_{1}$ & $0.5\left(F_{0} a\right)^{2}$ & 0.9 & \pm & 0.3 \\
$L_{2}$ & $1.1\left(F_{0} a\right)^{2}$ & 1.7 & \pm & 0.7 \\
$L_{3}$ & $10.4\left(F_{0} a\right)^{2}$ & -4.4 & \pm & 2.5 \\
$L_{4}$ & $0\left(F_{0} a\right)^{2}$ & 0 & \pm & 0.5 \\
$L_{5}$ & $0\left(F_{0} a\right)^{2}$ & 2.2 & \pm & 0.5 \\
$L_{6}$ & $0\left(F_{0} a\right)^{2}$ & 0 & \pm & 0.3 \\
$L_{7}$ & $0.9\left(F_{0} a\right)^{2}$ & -0.4 & \pm & 0.15 \\
$L_{8}$ & $2.6\left(F_{0} a\right)^{2}$ & 1.1 & \pm & 0.3 \\
$L_{9}$ & $-6.7\left(F_{0} a\right)^{2}$ & 7.4 & \pm & 0.2 \\
$L_{10}$ & $-6.7\left(F_{0} a\right)^{2}$ & -5.7 & \pm & 0.3 \\
\hline
\end{tabular}

Table 1: Comparison of the strong coupling expansion with experimental results from [4], [14]. The left column contains our results from the strong coupling expansion. The right column contains the experimental values $L_{i}^{r}\left(m_{\eta}\right)$. All numbers are in units of $10^{-3}$.

\section{Beyond the strong coupling regime}

We have chosen to express the entries in Table 1. in terms of a common factor $\left(F_{0} a\right)^{2}$ because of a point we will be making below, but the value of this quantity is also determined by the strong coupling expansion, from the $O\left(p^{2}\right)$ terms in the lattice chiral Lagrangian, and is given by $2.1 N$. With 3 colors the entries in the table come out about one order of magnitude higher than the values derived from experiment. We take this to be an indication that the strong coupling limit produces too tight a binding between quark and antiquark, which in turn leads to an unacceptably high value when $F_{0}$ is expressed in terms of $a^{-1}$ (or, equivalently, to too large a value for $a$ ). If we allowed ourselves to set $a^{-1} \approx F_{0}$, which would be a more reasonable scale for a strong coupling calculation, the theoretical predictions for several coefficients would compare rather well with the experimental results. Of course we should not make too much of this agreement, because we expect the values of the coefficients to depend on detailed dynamical features of the interactions which may not be well reproduced by the strong coupling approximation. What is much more important is the demonstration, through the strong coupling expansion, of the main point we wanted to make, namely that one can formulate an effective chiral Lagrangian on the lattice and that this can be the vehicle for the derivation of the parameters of the continuum chiral Lagrangian.

Another point that should be mentioned is that the expansion for small lattice momenta that we have used to derive the coefficients corresponds to using the lattice chiral Lagrangian in the tree approximation. We have calculated a few of the corrections that would be induced by one loop diagrams and have found these to be small. However, the same factor of $\left(F_{0} a\right)^{2}$ which we discussed above enters also as a coefficient in the denominator of the loop diagrams, so that the statement that the loop corrections are small should be taken with caution. They would become larger if one could assign to $F_{0} a$ the smaller value which we advocated 
above. Nevertheless, even if the loop corrections turned out to be large in a more realistic calculation, this would only represent a technical problem and not a conceptual difficulty for the whole approach. The very important fact is that, because we are working with a regularized theory, all physical quantities are well defined and finite. Moreover, since the lattice system also exhibits spontaneous breaking of chiral symmetry and Goldstone bosons in the limit of vanishing quark mass, it will always be possible to perform an expansion for small lattice momenta. The coefficients of such expansion are in any case well defined quantities. Thus the question is whether they can be calculated by a perturbative expansion of the chiral effective theory, which would be more convenient, or whether, failing such possibility, one will have to resort to numerical techniques. Nevertheless, even in the latter case, one would still be dealing with a bosonic system, and would therefore avoid the need of simulating the quantum fluctuations of fermionic variables, which represents today the major difficulty one faces in the implementation of QCD numerical simulations.

Finally, in order to obtain reliable values for the coefficients one should perform the calculation in the range of values of the lattice coupling constant $(\beta \approx 5.7-6$, including quark degrees of freedom in the simulation) where one witnesses the onset of scaling to the continuum. Such a calculation can at present only be done by numerical techniques. The way we envisage it could be carried out would be by assuming a sufficiently large set of couplings for the lattice effective theory and fixing them through the matching of an overcomplete set of expectation values. This is very similar to procedures commonly used in numerical studies of renormalization group transformations. The crucial point is that, because the lattice effective theory already accounts for the long range excitations of QCD, the matching should only require a reasonably small lattice size, of the order of the inverse $\rho$ mass. The two theories (the one derived from the original QCD Lagrangian and the effective theory) should produce exactly the same values for the observables on any lattice size, because they are mathematically equivalent. Working with a small lattice, we are confident, one would be able to fix the parameters of the lattice effective theory with a good degree of accuracy and would thus establish a solid base for the subsequent determination of the parameters of the continuum effective chiral Lagrangian.

Acknowledgments We would like to thank Richard Brower, Christophe Bruno, Sekhar Chivukula, Andrew Cohen, Gerhard Ecker, Vic Emery, Barry Holstein, Dimitris Kominis, Yue Shen, Jan Smit, Akira Ukawa and Steven Weinberg for useful and stimulating discussions.

\section{Appendix A. Goldstone bosons}

In this appendix, we will show that the fields $\pi_{x}$ are Goldstone bosons, corresponding to the spontaneous breaking of the chiral symmetry.

It was shown in [5], [6], for the case of no external sources on the hypercubical lattice, that the action in (39) exhibits spontaneous chiral symmetry breaking:

$$
U(n) \times U(n) \rightarrow U(n) .
$$

In that context, $n$ represented the number of color triplets of staggered fermions. Our situ- 
ation is somewhat different: we start from full-fledged fermions with Dirac, flavor and color indices and $n$ represents the number of flavors. Moreover, we have a different lattice structure.

We obtain the inverse propagator in momentum stas space for $n^{2}$ fields $\pi_{x}$ from expressions (49), (50), (56) and (57) by expanding to the quadratic order in powers of fields $\pi_{x}$ :

$$
\begin{aligned}
& D(p)=B\left[12-\sum_{v} \cos (p \cdot v)\right], \\
& B=\frac{4 N}{F_{0}^{2}}\left[\lambda_{0} F^{\prime}\left(\lambda_{0}\right)+\lambda_{0}^{2} F^{\prime \prime}\left(\lambda_{0}\right)\right] .
\end{aligned}
$$

We can rewrite the momentum dependent part of $D(p)$ in terms of momentum components $p_{\mu}$ :

$$
\begin{aligned}
\sum_{v} \cos (p \cdot v)=2 \quad & {\left[\cos \frac{p_{4}}{\sqrt{2}}\left(\cos \frac{p_{1}}{\sqrt{2}}+\cos \frac{p_{2}}{\sqrt{2}}+\cos \frac{p_{3}}{\sqrt{2}}\right)+\right.} \\
& \left.\cos \frac{p_{3}}{\sqrt{2}}\left(\cos \frac{p_{1}}{\sqrt{2}}+\cos \frac{p_{2}}{\sqrt{2}}\right)+\cos \frac{p_{1}}{\sqrt{2}} \cos \frac{p_{2}}{\sqrt{2}}\right] .
\end{aligned}
$$

By substituting this back into the expression (76) one can immediately see that the propagator $D^{-1}(p)$ has a massless pole $p_{4}=i m=0$ at the origin $\mathbf{p}=0$ for each of the $n^{2}$ fields contained in the matrix $\pi_{x}$.

\section{Appendix B. Lorentz covariance}

In this appendix we derive relation (69). From the definition (60):

$$
\begin{aligned}
\sum_{v} a_{v} b_{v} & =\sum_{i, j, \alpha} \frac{a_{i}+\alpha a_{j}}{\sqrt{2}} \frac{b_{i}+\alpha b_{j}}{\sqrt{2}} \\
& =\frac{1}{2} \sum_{i, j, \alpha}\left[a_{i} b_{i}+a_{j} b_{j}+\alpha\left(a_{i} b_{j}+a_{j} b_{i}\right)\right] .
\end{aligned}
$$

When summing over $\alpha= \pm 1$, terms proportional to $\alpha$ cancel and the others get a factor of 2:

$$
\sum_{v} a_{v} b_{v}=\sum_{i, j}\left(a_{i} b_{i}+a_{j} b_{j}\right)
$$

Finally, we perform the sum over $1 \leq i<j \leq 4$ and obtain:

$$
\sum_{v} a_{v} b_{v}=3 \sum_{\mu} a_{\mu} b^{\mu}
$$

Relation (70) can be proven in the same way.

\footnotetext{
${ }^{14}$ In this appendix, for simplicity we take $a=1$.
} 


\section{References}

[1] S. Weinberg, Phys. Rev. 166 (1968) 1568

[2] M. Gell-Mann, Physics 1 (1964) 63

[3] J. Gasser and H. Leutwyler, Ann. Phys. 158 (1984) 142

[4] J. Gasser and H. Leutwyler, Nucl. Phys. B 250 (1985) 465

[5] H. Kluberg-Stern, A. Morel, O. Napoly and B. Petersson, Nucl. Phys. B 190 (1981) 504

[6] N. Kawamoto and J. Smit, Nucl. Phys. B 192 (1981) 100

[7] B. M. Boghosian, Nucl. Phys. B (Proc. Suppl.) 30 (1993) 204

[8] H. Neuberger, Phys. Lett. B 199 (1987) 536

[9] W. Celmaster, Phys. Rev. D 26 (1982) 2955; Phys. Rev. D 28 (1983) 2076; Phys. Rev. Lett. 52 (1984) 403

[10] W. Celmaster and F. Krausz, Phys. Rev. D 28 (1983) 1527

[11] G. Bhanot, K. Bitar, U. M. Heller and H. Neuberger, Nucl. Phys. B 343 (1990) 467

[12] H. Georgi, Weak Interactions and Modern Particle Theory (Benjamin/Cummings, 1984)

[13] J. F. Donoghue, E. Golowich and B. R. Holstein, Dynamics of the Standard Model (Cambridge University Press, 1992)

[14] U. G. Meissner, Bern preprint BUTP-93/01

[15] B. Holdom, J. Terning and K. Verbeek, Phys. Lett. B 232 (1989) 351; Phys. Lett. B 245 (1990) 612; erratum: Phys. Lett. B 273 (1991) 549

[16] D. Ebert and H. Reinhardt, Nucl. Phys. B 271 (1986) 188

[17] G. Ecker, J. Gasser, A. Pich and E. de Rafael, Nucl. Phys. B 321 (1989) 311

[18] D. Espriu, E. de Rafael and J. Taron, Nucl. Phys. B 345 (1990) 22; erratum: Nucl. Phys. B 355 (1991) 278

[19] J. Bijnens, C. Bruno and E. de Rafael, Nucl. Phys. B 390 (1993) 501

[20] E. Brézin and D. J. Gross, Phys. Lett. B 97 (1980) 120

[21] I. Ichinose, Nucl. Phys. B 249 (1985) 715

[22] S. I. Azakov and É. S. Aliev, Sov. J. Nucl. Phys. 49 (1989) 875 